\title{
Involvement of SRPK1 in cisplatin resistance related to long non-coding RNA UCA1 in human ovarian cancer cells
}

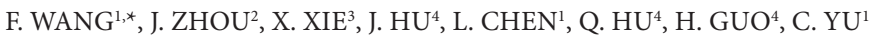 \\ ${ }^{1}$ Department of Gynecology, Shaannxi Provincial People's Hospital, Xi'an 710068, China; ${ }^{2}$ Department of Pediatrics, Second Affiliated Hospital of \\ Xian Jiaotong University College of Medicine, Xian 710054, China; ${ }^{3}$ Center for Clinical Laboratory, Shaanxi Provincial People's Hospital, Xian \\ 710068, China; ${ }^{4}$ Central Laboratory, Shaanxi Provincial People’s Hospital, Xian 710068, China
}

*Correspondence: fanwangfwre3@163.com

Received May 12, 2014 / Accepted November 24, 2014

\begin{abstract}
The therapeutic potential of cisplatin in ovarian cancer treatment is restricted by the occurrence of cellular resistance. We aimed to explore the role of SRPK1 in cisplatin resistance related to the long non-coding RNA UCA1 in ovarian cancer cell.

Totally, 24 ovarian cancer tissues and 16 normal tissues were used to assess the expression of UCA1 RNA. UCA1 stable transfected SKOV3 cells were established and the ability of cell migration, invasion and cisplatin resistance was assessed. The expression of SRPK1 and apoptosis pathway proteins was then assessed to explore the mechanism. In addition, SRPK1 knockdown cell line was also established and the effects of SRPK1 on cell migration, invasion and cisplatin resistance was evaluated.

Elevated expression of UCA1 RNA was identified in ovarian cancer tissues compared with normal tissues. Expression of UCA1 RNA in SKOV3 cells enhanced the cell migration, invasion and cisplatin resistance. Increased expression of SRPK1 and anti-apoptosis proteins were found in SKOV3/pcDNA-UCA1 cells. Knocking-down SRPK1 could partly rescue the effect of UCA1 expression on cell migration, invasion and cisplatin resistance in SKOV3 cells.

Elevated expression of UCA1 RNA was found in ovarian cancer tissues. UCA1 can improve the cell migration, invasion and induce cisplatin resistance. SRPK1 and apoptosis pathway proteins may be involved in the effect of UCA1.
\end{abstract}

Key words: UCA1, SRPK1, cisplatin resistance apoptosis pathway, ovarian cancer

Ovarian cancer is the second most commonly diagnosed gynecological cancer in the world, and causes more deaths per year than any other female reproductive system cancer [1]. More than 200,000 cases are newly diagnosed and 120,000 women die of ovarian cancer annually all over the world [2]. The chemotherapy based on platinum is active in ovarian cancer treatment. However, intrinsic or acquired cellular resistance to cisplatin is encountered regularly and severely limits the therapeutic potential of the drug [3]. Multiple biological processes, such as dose accumulation, metabolism, apoptosis, and DNA damage are suggested to be involved in the mechanisms of cellular drug resistance [4]. Considerable efforts have been taken to solve cisplatin resistance throughout the past three decades [5-7]. However, conquering this problem remains a critical goal for cancer therapy.

SRPK1 is a kinase which belongs to SR kinase family [8]. Through regulating the phosphorylation of SR splicing factors, SRPK1 can affect the pre-mRNA splicing and consequently gene expression [9]. Schenk et al [10] showed that activation of SRPK1 could induce cisplatin resistance in human ovarian carcinoma cell lines. Increasing attentions have been paid on the role of SRPK1 in cisplatin resistance $[10,11]$. In addition, the apoptosis resistance induced by anticancer drug treatment (such as cisplatin) has been suggested as another important mechanism in cellular drug resistance [4].

More and more studies have shown that abnormal expression of long non-coding RNA (lncRNA) is involved in tumor development and progression [12]. In a previous study, we obtained lncRNA UCA1 using Rapid Amplification of cDNA Ends (RACE) and found that the expression of lncRNA UCA1 was higher in bladder tumor tissues than in normal tissues [13]. However, the role of UCA1 in ovarian cancer has not clarified yet. Here, we tried to assess the expression of UCA1 and SRPK1 in ovarian cancer tissues together with normal tissues using RT-PCR and explore the role of UCA1 in cisplatin induced resistance. Our results might provide 
theoretical basis for chemotherapy selection in clinic and UCA1 was also suggested as a novel target related to cisplatin resistance.

\section{Materials and methods}

Ovarian tumor specimens. Flash frozen tissue specimens $(\mathrm{n}=40)$ were obtained from patients undergoing debulking surgery for ovarian cancer at People's Hospital of Shaanxi Providence, Shaanxi, China from January 2010 to January 2013. Among the specimens, epithelial ovarian cancer $(\mathrm{n}=$ 24) were obtained from primary lesion of patients without radio-chemotherapy while normal ovarian samples $(n=16)$ were obtained from patients undergoing hysterectomies for benign conditions. The pathological examination on all tissues was confirmed by two experienced physician. Written consent was provided by each patient and the whole protocol was approved by the Review Board of the hospital.

Cells. The human ovarian cancer cell line SKOV3 was maintained at $37^{\circ} \mathrm{C}$ and $5 \% \mathrm{CO}_{2}$ incubator in RPMI-1640 media with $10 \%$ fetal bovine serum, $100 \mathrm{U} / \mathrm{mL}$ penicillin, and $100 \mu \mathrm{g} / \mathrm{mL}$ streptomycin.

Reverse transcription PCR (RT-PCR) analysis. Total RNA extraction of tissue and cells were performed with Trizol (Life Tech, US) and the reverse transcription reaction were performed with ImProm II reverse transcriptase (Promega, US) according to the manufacturer's instructions. UCA1, SRPK1, $18 \mathrm{~S}$ rRNA specific sequences were amplified during 30 cycles of $30 \mathrm{~s}$ denaturing at $95^{\circ} \mathrm{C}, 60 \mathrm{~s}$ annealing at $57^{\circ} \mathrm{C}$, and 60 s extension at $72^{\circ} \mathrm{C}$, with the primers listed in Table 1 .

UCA1 expressed in SKOV 3 cells. The full length of UCA1 was expanded at an annealing temperature of $53^{\circ} \mathrm{C}$. After digested with BamHI and EcoRI, the PCR fragment was subcloned into pcDNA3.1 to construct the pcDNA-UCA1 plasmid. Transient transfection of cells with plasmid was performed with Lipofectamine 2000 (Life Tech, US). Twentyfour hours later, G418 (500 $\mu \mathrm{g} / \mathrm{mL})$ selection was processed for 3 weeks to get stable expressing cells. The characterization of the positive clone (SKOV3/pcDNA-UCA1) was confirmed by RT-PCR. The pcDNA3.1 without UCA1 fragment was used as negative control (SKOV3/pcDNA3.1).

RNA interference of SRPK1. The shRNA sequences of SRPK1 were obtained according to previous description [14]. SH1 and SH3, encoding shRNA targeting nucleotides 1171 to 1189 (GGTCAGTCATTCAGTGAACAA) and 647 to 655 (CAAGAAGATCCTAATGATTA), respectively, of the SRPK1
mRNA, were processed with annealing, subcloning into PRNATU6.1/Neo plasmid (GenScrpt Corp., Piscataway, NJ, US), plasmid expansion and media amount extraction. Transient transfection of cells with plasmid was performed with Lipofectamine ${ }^{\circ}$ 2000 (Life Tech, US) and 3 different batch of cells were used for knockdown efficacy examinations. Stable expressing cell lines were obtained by G418 (500 $\mu \mathrm{g} / \mathrm{mL})$ selection for 3 weeks. The expression of SRPK1 was confirmed by western-blot analysis.

Western-blot analysis. Cells were lysed in RIPA buffer (Beyotime, China), followed by high speed centrifugation and BCA quantification. Cellular protein was separated by SDSPAGE gel electrophoresis and then transferred onto PVDF membrane. After blocking, the blots were incubated with the antibodies to SRPK1 (BD, US), Bcl-2 (Cell Signaling Technology, US), BAX (Cell Signaling Technology, US), caspase-3 (Cell Signaling Technology, US), caspase-9 (Cell Signaling Technology, US). And $\beta$-Actin (Cell Signaling Technology) was used as a loading control. The appropriate HPR conjugated secondary antibodies were applied. The protein bands detected with Super Signal Ultra Chemiluminescent Substrate (Pierce, US) on chemiluminescence imager.

MTT assay. After preparing the single cell suspension, $4 \times 10^{3}$ cells in $100 \mu \mathrm{L}$ culture media were seeded in 96-well plate in quadruplicate overnight. MTT (Methyl thiazolylterazolium) was added in the cells for $4 \mathrm{hrs}$, and after formazan dye was dissolved with DMSO, the absorbance was read at $490 \mathrm{~nm}$ via a microplate reader (Molecular Device, US). All the experiments were performed independently for three times.

Clonogenic survival assay. Cells $\left(5 \times 10^{2}\right)$ were seeded in 6-well plate and incubated with complete media (RPMI1640 with $10 \%$ FBS) and $500 \mu \mathrm{g} / \mathrm{mL}$ G418 for 14 day. After removing the media, cells were washed with PBS, fixed with 95\% ethanol for $30 \mathrm{~min}$ and stained with Giemsa for $15 \mathrm{~min}$. Colonies with $>50$ cells were counted under microscope. Percentage cell survival was expressed relative to untreated control.

2-D Scratch assay. Cells $\left(3 \times 10^{5}\right)$ were seeded in 6-well plate and were allowed to grow until $90 \%$ confluence was reached. Then the cells were grown in $0.2 \%$ FBS RPMI 1640 media overnight for serum starving and a scratch was made by using the $200 \mu \mathrm{L}$ pipette tip. Debris was removed with PBS and then the culture was re-fed with fresh medium. The photos were taken at $0 \mathrm{~h}$ and $24 \mathrm{~h}$ under a microscopy and the relative ratio of the recovery wound areas were calculated according to the images.

3-D Migration and invasion assay. Cells $\left(5 \times 10^{5}\right)$ were seeded in triplicate in upper chamber of the Millicell $(8 \mu \mathrm{m}$ pore

Table 1. Primer sequences used in the study

\begin{tabular}{lll}
\hline Name & Forward primer & Reverse primer \\
\hline UCA1 & 5'-CTCTCCATTGGGTTCACCATTC-3' & 5'-GCGGCAGGTCTTAAGAGATGAG-3' \\
SRPK1 & 5'-TAA CGG ACC ACT GGA CAA CAA A-3' & 5'TTC CTG CGA CCA CTC ATA CTT C 3' \\
18SrRNA & 5'-CAGCCACCCGAGATTGAGCA-3' & 5'-TAGTAGCGACGGGCGGTGTG-3' \\
UCA1 (full length) & 5'-CGGGATCCTGACATTCTTCTGGACAATGAG-3’' & 5'-CCGGAATTCGCATATTAGCTTTAATGTAGGTGGC-3’ \\
\hline
\end{tabular}


diameter) which was pre-coated with Matrigel (Becton Dickinson Lab ware, Bedford, MA, US). After the lower chamber of the Millicell was added with $900 \mu \mathrm{L}$ complete medium (RPMI 1640 with $20 \%$ FBS), the Millicell was incubated at $37^{\circ} \mathrm{C}$ and $5 \% \mathrm{CO}_{2}$ for $24 \mathrm{hrs}$. The Matrigel was then removed by cotton tip, fixed with $95 \%$ ethanol for $30 \mathrm{~min}$, stained with Giemsa for $15 \mathrm{~min}$. The membrane was checked with microscopy. The migration assay was similar with invasion assay. The cells were seeded in uncoated Millicell chamber and the microscopy assessment was performed after incubation for $12 \mathrm{hrs}$.

Cell viability assay treated with cisplatin. Cells $\left(1 \times 10^{4}\right)$ were seeded in quadruplicate in 96-well plate for overnight adherence. Then the cells were treated with series concentration of cisplatin $(0,2.5,5,10,20,40,80 \mu \mathrm{M}$; Cisplatin was dissolved in $0.9 \% \mathrm{NaCl}$ ) for $48 \mathrm{hrs}$. Cell viability was determined by MTT assay at $490 \mathrm{~nm}$ wavelength. Then the $\mathrm{IC}_{50}$ (the concentration at which the curve passes through the $50 \%$ inhibition level) was calculated.

Statistical analysis. All statistical analyses were performed using the SPSS13.0 software (SPSS Inc. Chicago, IL, US). The results were presented as means \pm standard deviation (SD).
Two-tailed Student's t-test was used to examine the differences between groups. $\mathrm{P}<0.05$ was considered as statistically significant.

\section{Results}

The expression of UCA1 RNA and SRPK1 mRNA in ovarian tissues. We detected the expression of UCA1 and SRPK1 in the 24 ovarian epithelial cancer tissues and 16 normal ovarian tissues, and found that the expressions of UCA1 RNA and SRPK1 mRNA in ovarian cancer tissues were comparatively higher, while no significant expression of UCA1 and SRPK1 were detected in normal ovarian tissues (Figure 1A).

The effect of UCA1 RNA expression on SKOV 3 migration and invasion and related mechanisms.

Establishment of SKOV3/pcDNA-UCA1 cells. After constructing of pcDNA-UCA1, the stable expressing cell lines with or without UCA1 RNA expression were established. Positive control was confirmed by RT-PCR and the result showed that a length of 1442 bps UCA1 RNA
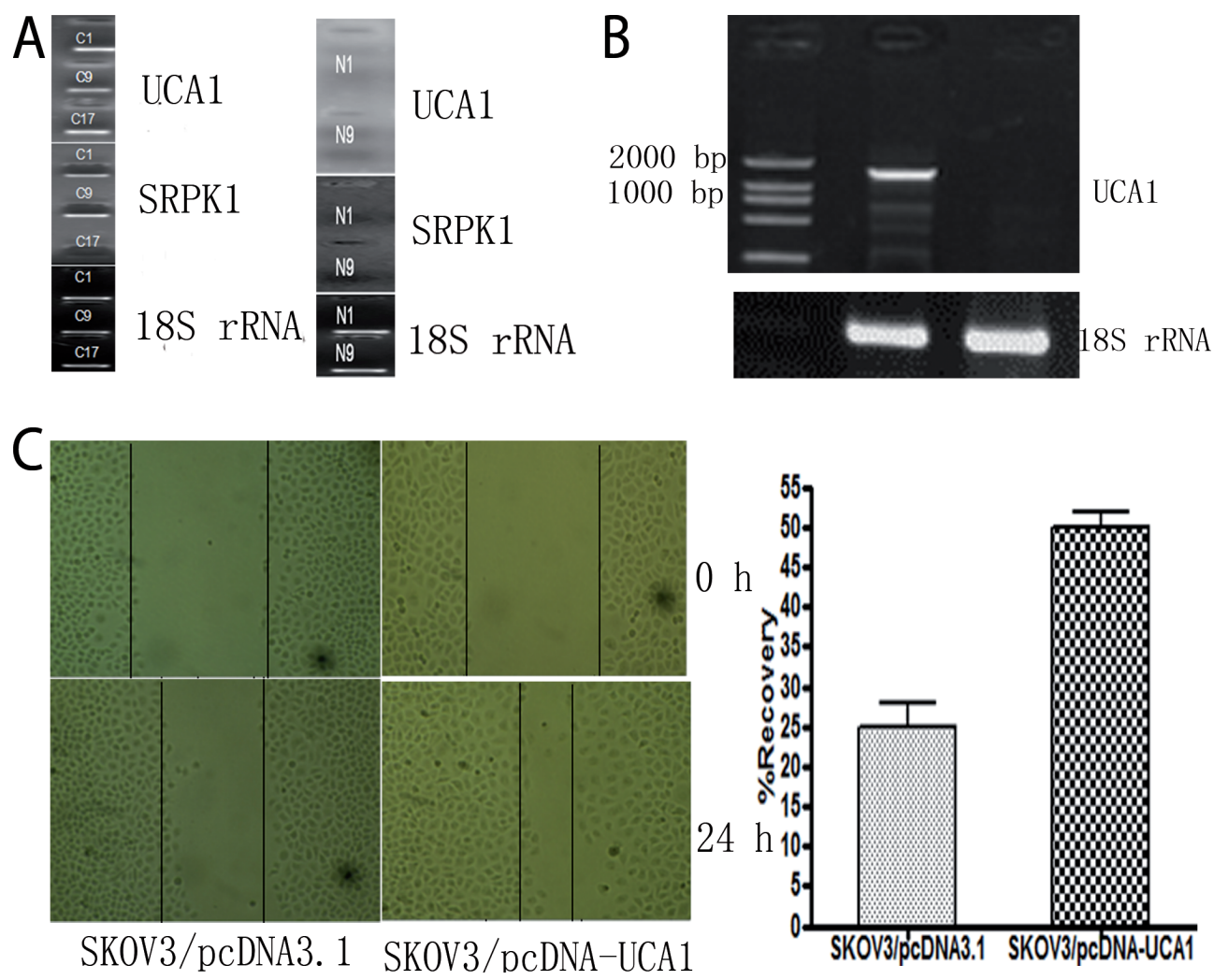

Figure 1. The expression of UCA1 RNA and SRPK1 mRNA in human tissues, characterization and scratch assay of SKOV3/pcDNA-UCA1 cells and SKOV3/ pcDNA 3.1 control cells. A. Elevated expression of UCA1 RNA and SRPK1 mRNA were found in 24 human ovarian cancer tissues (C1-C24) compared with 16 normal ovarian tissues (N1-N16). The expression of $18 \mathrm{~S}$ rRNA was used as internal control and no difference was found between samples; B. Established SKOV3/pcDNA-UCA1 cells and the expression of UCA1 RNA were identified by RT-PCR. SKOV3/pcDNA3.1 cells were used as negative control. C. Scratch assay with using SKOV3/pcDNA-UCA1 and control cells. Images were captured immediately at $0 \mathrm{~h}$ and $24 \mathrm{~h}$ after wounding. Representative microscopic images were shown (left, 40×Magnification), scratch-wound edges were marked using black lines. The quantification of migration (percentage of recovery) was shown by bar graph. The results represented the means \pm SD value from three independent experiments $\left(\right.$ right, $\left.{ }^{*} p<0.01\right)$ 
was expanded from SKOV3/pcDNA-UCA1 cells while no UCA1 was found in negative control SKOV3/pcDNA 3.1 cells (Figure 1B).

2-D and 3-D migration and invasion assay. The scratch assay suggested that cell migration ability of SKOV3/pcDNAUCA1 significantly increased compared with that of SKOV3/ pcDNA 3.1 (Figure 1C). The 3-D migration and invasion assay with Millicell chamber showed that the migration and invasion abilities were significantly increased in SKOV3/ pcDNA-UCA1 cells compared with SKOV3/pcDNA 3.1 cells (Figure 2A).

SKOV3/pcDNA-UCA1 cells viability assay treated with cisplatin. The cell viability assay treated with cisplatin was performed with SKOV3/pcDNA-UCA1 and SKOV3/pcDNA 3.1 cells by MTT. Higher cell viability was found in SKOV3/ pcDNA-UCA1 cells. The $\mathrm{IC}_{50}$ of SKOV3/pcDNA-UCA1 cells increased by 2.41 times compared with that of SKOV3/pcDNA 3.1 cells (Figure $2 \mathrm{~B}$ ).

Western blot analysis of SRPK1 and apoptosis pathway. The expressions of SRPK1, Bcl-2, Bax, Caspase-3 and Caspase-9 in SKOV3/pcDNA-UCA1 and SKOV3/pcDNA 3.1 cells were also checked, and there were increased expressions of SRPK1 and $\mathrm{Bcl}-2$ and decreased expressions of Bax, Caspase- 3 and Caspase-9 in SKOV3/pcDNA-UCA1 cells (Figure 2C).

The effect of SRPK1 knockdown on SKOV3 cells.

Knockdown cell line establishment. The knockdown efficacy of pRNAT-SH1 and pRNAT-SH3 were firstly examined by western-blot. The results showed that SKOV3/ pRNAT-SH3 exerted more obvious knock down effect on SRPK1 (Figure 3A1). The knockdown effect of pRNAT-SH3 on SRPK1 in transient transfection cells was shown in Figure 3A2.

The proliferation, colongenic, migration, invasion and cisplatin resistance abilities of SRPK1 knockdown cells. The results of MTT assay showed that decreased proliferation was found in SKOV3/pRNAT-SH3 (Figure 3B). The clonogenic ability of SKOV3/pRNAT-SH3 significantly decreased compared to that of SKOV3/pRNAT-U6.1 from Day 3 to Day 6 (Figure 3C). The 3-D migration and cell invasion assay showed that the ability of cell migration and invasion decreased in SKOV3/pRNAT-SH3 cells than that in SKOV3/pRNAT-U6.1 cells (Figure 4A).
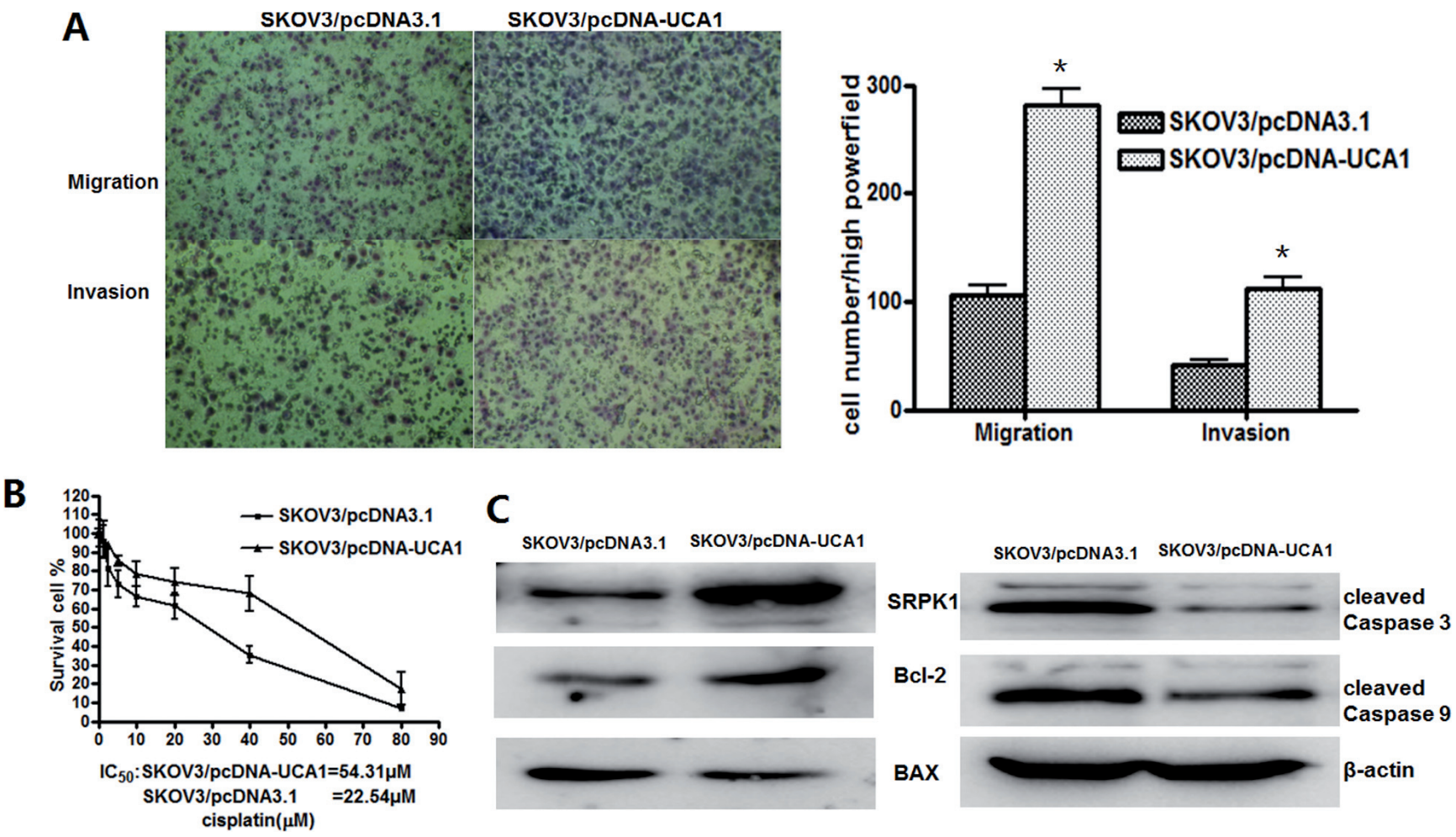

Figure 2. Migration, invasion, cisplatin resistance assay and related mechanism analyzed by using SKOV3/pcDNA-UCA1 cells and SKOV3/pcDNA 3.1 control cells. A. Increasing migration and invasion were found in SKOV3/pcDNA-UCA1 cells compared with control. Representative images on migration and invasion in SKOV3/pcDNA-UCA1 cells and SKOV3/pcDNA3.1 cells (left). The quantification of migration and invasion were shown by bar graph. The results represented total numbers of migration or invasion cells in 10 random fields at $400 \times$ (right). Significant difference was found in SKOV3/pcDNA-UCA1 cells compared with SKOV3/pcDNA3.1 cells $\left({ }^{*} \mathrm{p}<0.05\right)$; B. Cisplatin resistance assay. The IC ${ }_{50}$ increased 2.41 times in SKOV3/pcDNA-UCA1 cells compared with the SKOV3/pcDNA3.1 cells; C. The increased expression of SRPK1 and Bcl-2 and decreased expression of BAX, cleaved Caspase- 3 and cleaved Caspase-9 were found in SKOV3/pcDNA-UCA1 cells compared with SKOV3/ pcDNA3.1 cells. 
SKOV3/pRNAT-SH3 cell viability assay treated with cisplatin. The cell viability assay treated with cisplatin was performed in SKOV3/pRNAT-SH3 and SKOV3/pRNAT-U6.1 cells by using MTT. Lower cell viability was found in SKOV3/ pRNAT-SH3 cells. The $\mathrm{IC}_{50}$ of SKOV3/pRNAT-SH3 cells was decreased by 2.64 times than that of SKOV3/pRNAT-U6.1 cells (Figure 4B).

The analysis of SRPK1 and apoptosis pathway by western blot. To explore the mechanism, we analyzed the expression of Bcl-2, Bax, Caspase-3 and Caspase-9 in SKOV3/pRNAT-SH3 and SKOV3/pRNAT-U6.1 cells and found that decreased expression of $\mathrm{Bcl}-2$ and increased expressions of Bax, Caspase- 3 and Caspase-9 in SKOV3/pRNAT-SH3 cells (Figure 4C).

\section{Discussion}

The lncRNA UCA1 was firstly cloned in our lab using SMAT-RACE from the bladder cancer cell line BLZ-211 [13], and UCA1 RNA showed an expression pattern of increased expression at early stage of human embryonic development, differential expression at 28 week of embryonic development, and no expression in normal adult tissues, while the expression increased in bladder cancer tissues. In addition, the increasing expression of UCA1 RNA comparing to the normal or para-carcinoma tissues was also found in breast cancer, liver cancer, thyroid cancer, cervical cancer, lung cancer, esophagus cancer, and gastric cancer [15]. In this study, we also observed obvious expression of UCA1 RNA in ovarian cancer tissues, while there was no expression in normal tissues. These results suggest UCA1 RNA may exert a critical role in the development and progression of ovarian cancer.

It was reported that the abilities of cell proliferation, cisplatin resistance, invasion and migration increased in bladder cancer cell line [16]. Wang et al found that overexpression of UCA1a (also named as CUDR) in bladder cancer cells would cause these increases, as well as the decrease of cell apoptosis [17]. Wing et al found similar changes in A431 squamous cancer cells [18]. Nevertheless, they failed to explain the accurate mechanisms [19]. In addition, Wu et al reported that the Ets-2 binding site located in the promoter region of UCA1 and they proposed Ets- 2
A

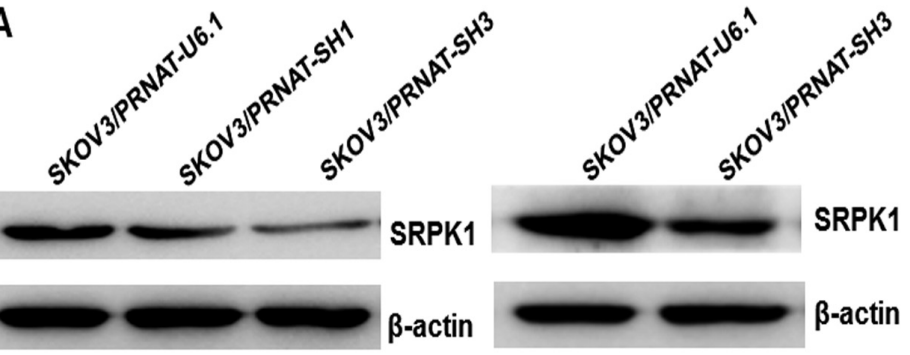

C SKOV3/PRNAT-U6.1 SKOV3/PRNAT-SH3

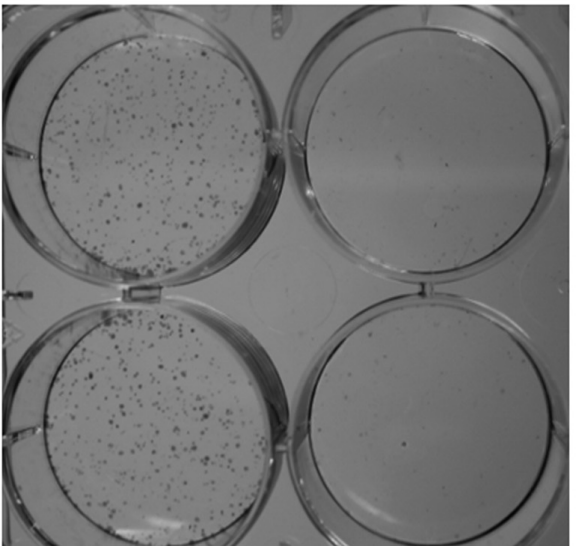

B
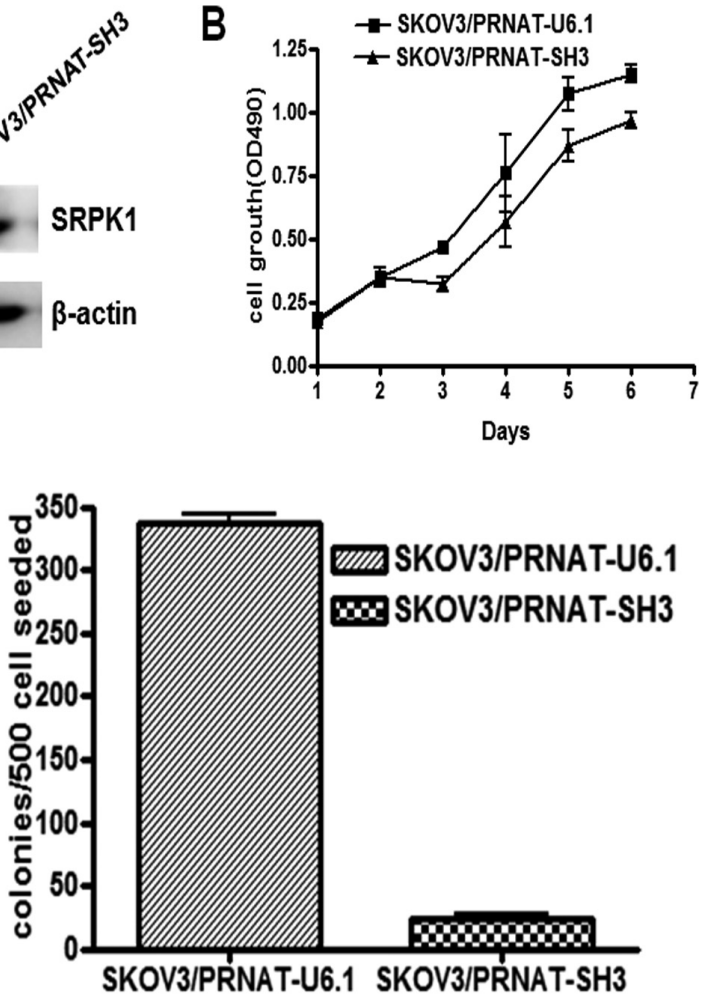

Figure 3. Knocking down SRPK1 in SKOV3 cells; Cisplatin resistance and clonogenic survival assay by using SRPK1 knockdown cells and control cells. A. Knock down of SRPK1 by RNAi. SKOV3/PRNAT-U6.1 cells were used as a negative control and $\beta$-actin was used as a loading control. (A) Western blot analysis indicated SRPK1 protein levels are suppressed in SKOV3/PRNAT-SH1 and SKOV3/PRNAT-SH3 transient transfection of cells compared to that of SKOV3/PRNAT-U6.1 cells. $\left(\mathrm{A}_{2}\right)$ Western blot analysis indicated SRPK1 protein levels are suppressed in SKOV3/PRNAT-SH3 stable transfection of cells compared to that of SKOV3/PRNAT-U6.1 cells. $\beta$-actin was used as loading control. B. MTT assay was used to assess the effect of SRPK1 knockdown on SKOV3/PRNAT-SH3 and SKOV3/PRNAT-U6.1 cells . The data were from three independent experiments. Significant difference was found from Day 3 to Day 6 (p<0.05). C. Clonogenic survival assay of SKOV3/PRNAT-SH3 and SKOV3/PRNAT-U6.1 cells. Representative images of cultures were shown. Significant decreased colony formation was found in SKOV3/PRNAT-SH3 cells compared with SKOV3/PRNAT-U6.1 cells $\left({ }^{*}\right.$ p $\left.<0.001\right)$. 
regulates apoptosis process by regulating the expression of UCA1 [20]. Therefore, UCA1 may indirectly regulate the cell apoptosis. The cisplatin resistance of ovarian cancer is acknowledged to be the main cause of tumor recurrence and the failure of chemotherapy [21]. The mechanism of cisplatin resistance includs dose accumulation of the drug, metabolism, apoptosis and DNA damage, and it is a complicateed process of multi-factor, multilevel and multi-gene [4]. Most recently, Fan et al showed that UCA1 could induce cisplatin resistance in bladder cancer cells by regulating Wnt signaling [22]. Here, we established SKOV3 cell lines expressing UCA1 RNA and found that cell abilities of migration, invasion and cisplatin resistance increased.

SRPK1 was proved to be involved in cisplatin resistance $[23,24]$, therefore, we analyzed the association between UCA1 RNA and SRPK1 during cisplatin resistance. In UCA1 expression cell line SKOV3/pcDNA-UCA1, we found increased expression of SRPK1 and Bcl-2, as well as decreased expression of Bax, Caspase-3 and Caspase-9. SRPK1 is specific kinase belonging to SR family. It can specifically phosphorylate the SR splice factor and regulate the gene expression by alternative splicing of pre-mRNA of target gene [9]. Hayes et al found decreased expression of SRPK1 in pancreas, colon and breast cancer could lead to increasied and decreased expression of $\mathrm{Bcl}-2$ and Bax respectively. The decreasing proliferation and increasing apoptosis of cells were also found [25], along with the increased sensitivities to Gemcitabine and Cisplatin [14, 25]. Furthermore, Fan et al also suggested UCA1 overexpression could result in the increased expression of SRPK1 [22]. Here, we employed RNAi to decrease the expression of SRPK1 and found that increased expression of Bcl-2 and decreased expression of Bax, Caspase- 3 and Caspase- 9 after down-regulating the expression of SRPK1. In addition, we also found the increasing abilities on cell proliferation, migration and invasion after SRPK1 knockdown.

In conclusion, we suggest that SRPK1 may result in increased cell proliferation, and decreased apoptosis in ovarian cancer cells and induce the cisplatin resistance and UCA1 might activate SRPK1 in the ovarian cancer . Our results might add novel insight on cisplatin resistance and provide a novel molecular target to the treatment.
A

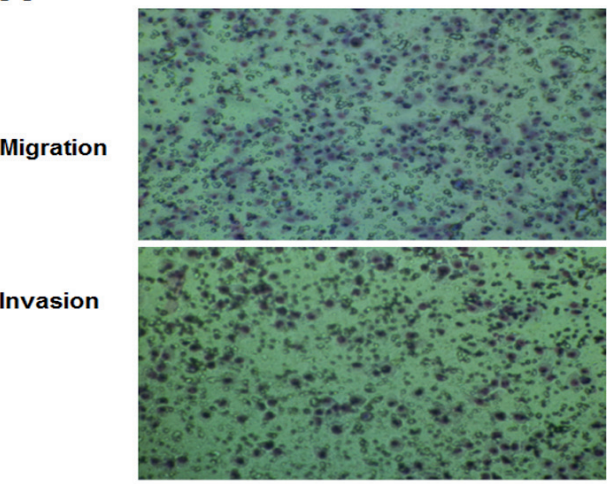

B

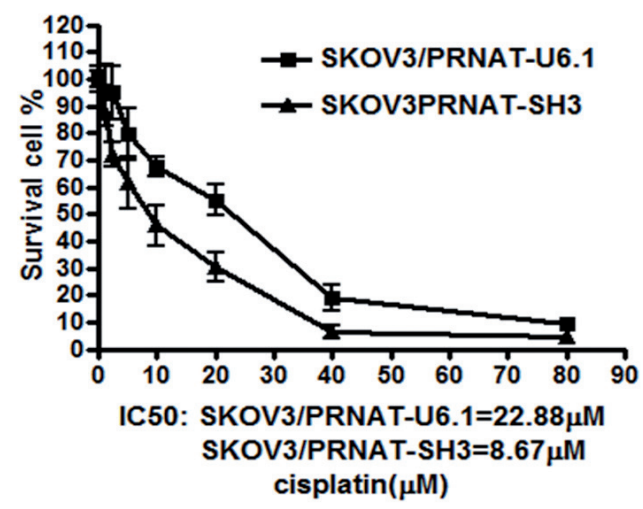

SKOV3/PRNAT-SH3

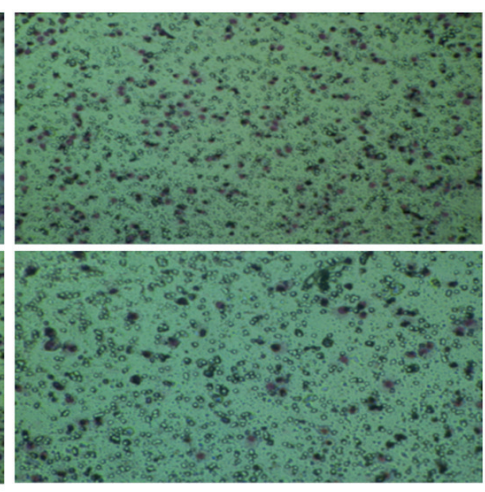

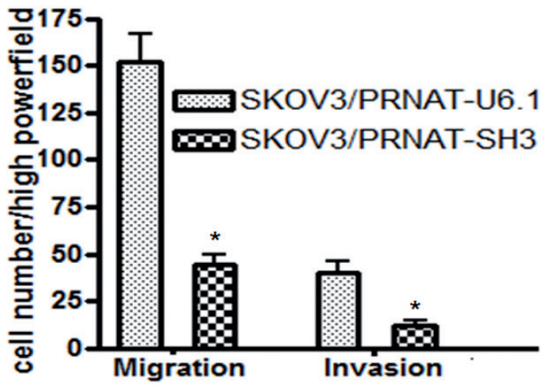

C
SKOV3/PRNAT-SH3

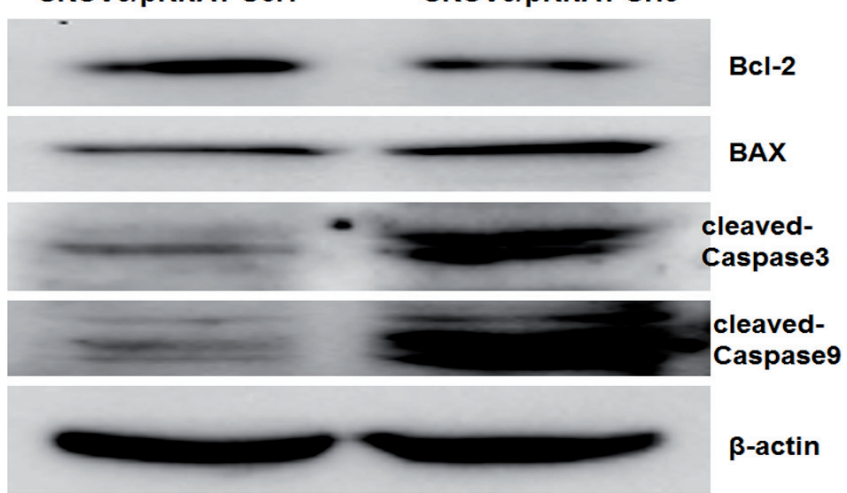

Figure 4. Influence of SRPK1 knocking down. A. Migration, invasion, cisplatin resistance assay and related mechanism analyses by using SKOV3/ PRNAT-SH3 cells and SKOV3/PRNAT-U6.1 control cells. Significant decreasing in migration and invasion of SKOV3/PRNAT-SH3 cells were found after SRPK1 knocking down. Representative images of cell migration and invasion were shown on left and quantification of cell number (Total cell number of 10 random selected fields at $400 \times$ magnification) was shown on right by bar graph $\left({ }^{*} p<0.01\right)$. B. the SKOV3/PRNAT-U6.1 cells. C. The decreased expression of Bcl-2 and increased expressions of BAX, cleaved Caspase- 3 and cleaved Caspase-9 were found in SKOV3/PRNAT-SH3 cells by western blot analysis. 
Acknowledgements: The Project was supported by a Grant from the National Natural Science Foundation of China (Program No. 30901600); The Project also Supported by Natural Science Basic Research Plan in Shaanxi Province of China (Program No. 2013JQ4003)

\section{References}

[1] PERMUTH-WEY J, SELLERS TA. Epidemiology of ovarian cancer. Methods Mol Biol. 2009; 472: 413-437. doi: 10.1007/978-1-60327-492-0_20.http://dx.doi.org/10.1007/9781-60327-492-0 20

[2] HUNN J, RODRIGUEZ GC. Ovarian cancer: etiology, risk factors, and epidemiology. Clin Obstet Gynecol. 2012; 55: 3-23. http://dx.doi.org/10.1097/GRF.0b013e31824b4611

[3] FLOREA AM, BUSSELBERG D. Cisplatin as an anti-tumor drug: cellular mechanisms of activity, drug resistance and induced side effects. Cancers (Basel). 2011; 3: 1351-1371. doi: cancers3011351 [pii]

[4] GALLUZZI L, SENOVILLA L, ViTALE I, MICHELS J, MARTINS I et al. Molecular mechanisms of cisplatin resistance. Oncogene. 2012; 31: 1869-1883. doi: onc2011384 [pii]

[5] KARTALOU M, ESSIGMANN JM. Mechanisms of resistance to cisplatin. Mutation Research/Fundamental and Molecular Mechanisms of Mutagenesis. 2001; 478: 23-43. http://dx.doi. org/10.1016/S0027-5107(01)00141-5

[6] SHEN D-W, POULIOT LM, HALL MD, GOTTESMAN MM. Cisplatin resistance: a cellular self-defense mechanism resulting from multiple epigenetic and genetic changes. Pharmacol Rev. 2012; 64: 706-721. http://dx.doi.org/10.1124/ pr.111.005637

[7] SIDDIK ZH. Cisplatin: mode of cytotoxic action and molecular basis of resistance. Oncogene. 2003; 22: 7265-7279. http:// dx.doi.org/10.1038/sj.onc.1206933

[8] MANLEY JL, TACKE R. SR proteins and splicing control. Genes Dev. 1996; 10: 1569-1579. http://dx.doi.org/10.1101/ gad.10.13.1569

[9] GRAVELEY BR. Sorting out the complexity of SR protein functions. RNA. 2000; : 1197-1211.

[10] SCHENK PW, BOERSMA AW, BRANDSMA JA, DEN DULK $\mathrm{H}$, BURGER $\mathrm{H}$ et al. SKY1 is involved in cisplatin-induced cell kill in Saccharomyces cerevisiae, and inactivation of its human homologue, SRPK1, induces cisplatin resistance in a human ovarian carcinoma cell line. Cancer Res. 2001; 61: 6982-6986.

[11] GOUT S, BRAMBILLA E, BOUDRIA A, DRISSI R, LANTUEJOUL $S$ et al. Abnormal expression of the pre-mRNA splicing regulators SRSF1, SRSF2, SRPK1 and SRPK2 in non small cell lung carcinoma. PLoS One. 2012; 7: e46539. http:// dx.doi.org/10.1371/journal.pone.0046539

[12] DENG G, SUI G. Noncoding RNA in oncogenesis: A new era of identifying key players. International journal of molecular sciences. 2013; 14: 18319-18349. http://dx.doi.org/10.3390/ ijms140918319

[13] WANG F, LI X, XIE X, ZHAO L, CHEN W. $<$ i $>$ UCA $1<$ i $>$, a non-protein-coding RNA up-regulated in bladder carcinoma and embryo, influencing cell growth and promoting invasion. FEBS letters. 2008; 582: 1919-1927. http://dx.doi. org/10.1016/j.febslet.2008.05.012

[14] HAYES GM, CARRIGAN PE, BECK AM, MILLER LJ. Targeting the RNA splicing machinery as a novel treatment strategy for pancreatic carcinoma. Cancer Res. 2006; 66: 3819-3827. http://dx.doi.org/10.1158/0008-5472.CAN-05-4065

[15] XIE XJ, LI X, WANG F, CHEN W. [Cellular localization and tissue expression pattern of UCA1, a non-coding RNA]. Nan Fang Yi Ke Da Xue Xue Bao. 2010; 30: 57-60.

[16] ALI AY, FARRAND L, KIM JY, BYUN S, SUH JY et al. Molecular determinants of ovarian cancer chemoresistance: new insights into an old conundrum. Ann N Y Acad Sci. 2012; 1271: 58-67. http://dx.doi.org/10.1111/j.1749-6632 $.2012 .06734 . \mathrm{x}$

[17] WANG Y, CHEN W, YANG C, WU W, WU S et al. Long non-coding RNA UCA1a (CUDR) promotes proliferation and tumorigenesis of bladder cancer. Int J Oncol. 2012; 41: 276.

[18] CO NN, TSANG WP, TSANG TY, YEUNG CLA, YAU PL ET AL. AF1q enhancement of $\gamma$ irradiation-induced apoptosis by up-regulation of BAD expression via NF- $\kappa B$ in human squamous carcinoma A431 cells. Oncology reports. 2010; 24: 547-554.

[19] TSANG WP, WONG TW, CHEUNG AH, KWOK TT. Induction of drug resistance and transformation in human cancer cells by the noncoding RNA CUDR. Rna. 2007; 13: 890-898. http://dx.doi.org/10.1261/rna.359007

[20] WU W, ZHANG S, LI X, XUE M, CAO S et al. Ets-2 regulates cell apoptosis via the Akt pathway, through the regulation of urothelial cancer associated 1, a long non-coding RNA, in bladder cancer cells. PLoS One. 2013; 8: e73920. doi: 10.1371/ journal.pone.0073920 http://dx.doi.org/10.1371/journal. pone. 0073920

[21] CHIEN J, KUANG R, LANDEN C, SHRIDHAR V. Platinum-Sensitive Recurrence in Ovarian Cancer: The Role of Tumor Microenvironment. Front Oncol. 2013; 3: 251. doi: 10.3389/fonc.2013.00251. http://dx.doi.org/10.3389/ fonc. 2013.00251

[22] FAN Y, SHEN B, TAN M, MU X, QIN Y et al. Long non-coding RNA UCA1 increases chemoresistance of bladder cancer cells by regulating Wnt signaling. FEBS J. 2014; 281: 1750-1758. doi: 10.1111/febs.12737. http://dx.doi.org/10.1111/febs.12737

[23] ODUNSI K, MHAWECH-FAUCEGLIA P, ANDREWS C, BECK A, AMUWO O et al. Elevated expression of the serinearginine protein kinase 1 gene in ovarian cancer and its role in Cisplatin cytotoxicity in vitro. PLoS One. 2012; 7: e51030. http://dx.doi.org/10.1371/journal.pone.0051030

[24] SCHENK PW, STOOP H, BOKEMEYER C, MAYER F, STOTER G et al. Resistance to platinum-containing chemotherapy in testicular germ cell tumors is associated with downregulation of the protein kinase SRPK1. Neoplasia (New York, NY). 2003; 6: 297-301. http://dx.doi.org/10.1593/neo.03406

[25] HAYES GM, CARRIGAN PE, MILLER LJ. Serine-arginine protein kinase 1 overexpression is associated with tumorigenic imbalance in mitogen-activated protein kinase pathways in breast, colonic, and pancreatic carcinomas. Cancer Res. 2007; 67: 2072-2080. http://dx.doi.org/10.1158/0008-5472.CAN$\underline{06-2969}$ 